\title{
CONFLUENCE OF INDIRECTION REDUCTIONS IN GRAPH REWRITE SYSTEMS
}

\author{
P.M. VAN DEN BROEK \\ Department of Computer Science, Twente University, P.O. Box 217, 7500 AE Enschede, The Netherlands \\ Communicated by W.L. Van der Poel \\ Received 28 November 1987
}

Keywords: Confluence property, indircetion graph, graph rewritc system

\section{Introduction}

Many graph rewrite systems use graphs with indirection nodes; an indirection node of a graph is a node with a single successor which only purpose is to redirect its incoming arcs to its successor. For instance, graph rewrite systems used in implementations of functional languages have indirection nodes [3,5]. Another example can be found in [1]. The process of transforming a graph by redirecting an incoming arc of an indirection node to the successor of the indirection node is called indirection reduction.

A useful property of many rewrite systems is the confluence property; for instance, it implies uniqueness of normal forms. To prove the confluence property of a graph rewrite system with indirection reductions one can, for instance, use results of Van den Broek and Van der Hoeven [6], or Rosen [4]. In either case one has to prove the confluence property for the graph rewrite system which has indirection reductions only. This is the subject of this paper.

The confluence property for indirection reductions does not hold for concrete graphs, as one can see by looking at the simple example of a graph with two nodes which are both indirection nodes and have each other as successor. So we have to consider abstract graphs, i.e., isomorphism classes of concrete graphs, which explains why the problem is nontrivial.
Note that the problem becomes trivial when one only considers acyclic graphs; this solution was chosen by Ehrig and Rosen [2].

This paper is organised as follows. The next section contains the necessary definitions and notation conventions which are used in this paper. In Section 3 we introduce equivalence relations on nodes and arcs of indirection graphs and prove a number of properties of these. In Section 4 we prove the confluence of a graph rewrite system which has only indirection reductions.

\section{Definitions and notation}

A rewrite system is a tuple $\langle B, \Rightarrow\rangle$, where $B$ is a set and $\Rightarrow$ is a binary relation on $B$.

Here we consider the case where $B$ is the set of indirection graphs. An indirection graph $G$ (we drop the prefix 'indirection' from now on) is a 5-tuple $\langle N, A, S, T, I\rangle$, where:

- $N$ is a finite set; its elements are called the nodes of $G$,

- $A$ is a finite set; its elements are called the arcs of $G$,

- $S$ is a mapping from $A$ lo $N$; it associates $t o$ each arc from $A$ its source in $N$,

- $T$ is a mapping from $A$ to $N$; it associates to each arc from $A$ its target in $N$,

- $I$ is a subset of $N$; its elements are called indirection nodes, 
such that the outdegree of each $x \in I$ is equal to 1 , i.e., for each $x \in I$ we have

$|\{a \in A \mid S(a)=x\}|=1$.

With each graph $G$ we associate a mapping $\operatorname{succ}_{G}: I \rightarrow N$ defined by $\operatorname{succ}_{G}(n)=T(a)$, where $a$ is the unique element of $A$ with $S(a)=n$. We denote $\operatorname{succ}_{G}(n)=m$ by $n \rightarrow_{G} m$, and the reflexive transitive closure of $\rightarrow_{G}$ by $\rightarrow_{G}^{*}$.

The binary relation $\Rightarrow$ on $B$ is given by: If $G$ and $G^{\prime}$ are graphs and $G=\langle N, A, S, T, I\rangle$, then $G \Rightarrow G^{\prime}$ if and only if $G=\left\langle N, A, S, T^{\prime}, I\right\rangle$ and there exists an $a \in A$ with $T(a) \in I$ such that $T^{\prime}$ satisfies

$T^{\prime}(x)=T(x)$ for each $x$ of $A$ with $x \neq a$,

$T^{\prime}(a)=\operatorname{succ}_{G}(T(a))$.

Informally, $G \Rightarrow G^{\prime}$ if and only if $G^{\prime}$ can be obtained from $G$ by giving an arc of $G$ whose target is an indirection node $b$ the new target $\operatorname{succ}_{G}(b)$. The process of actually replacing $G$ by $G^{\prime}$ is called an indirection reduction.

The reflexive transitive closure of $\Rightarrow$ is denoted by $\Rightarrow^{*}$.

The aim of this paper is to show that this rewrite system is confluent, i.e., if $G \Rightarrow^{*} G 1$ and $G \Rightarrow^{*} G 2$ for some graphs $G, G 1$, and $G 2$, then there exist graphs $G 1^{\prime}$ and $G 2^{\prime}$ which are isomorphic such that $G 1 \Rightarrow^{*} G 1^{\prime}$ and $G 2 \Rightarrow^{*} G 2^{\prime}$.

\section{Equivalence relations}

In order to prove confluence of indirection reductions in the next section we shall define in this section equivalence relations on the sets of nodes and arcs of a graph and derive a number of propertics of these.

In the remainder of this section, $G$ will be the graph $\langle N, A, S, T, I\rangle$.

We shall consider the equivalence relation $\propto_{G}$ on $N$ generated by the function $\operatorname{succ}_{G}$; so, $n \in N$ and $m \in N$ are equivalent $\left(n \propto_{G} m\right.$ ) if and only if these exist an integer $k \geqslant 1$ and $k$ elements $n_{1}$, $n_{2}, \ldots, n_{k}$ of $N$ such that

$$
\begin{aligned}
& m=n_{1}, \\
& n=n_{k}, \\
& n_{i} \rightarrow_{G} n_{i+1} \text { or } n_{i+1} \rightarrow_{G} n_{i} \\
& \quad \text { for all } i=1,2, \ldots, k-1 .
\end{aligned}
$$

On $A$ we shall consider the equivalence relation $\approx_{G}$ defined by

$a \approx_{G} b \Leftrightarrow T(a) \propto_{G} T(b)$.

The usefulness of these equivalence relations stems from the fact that they are invariant for indirection reductions.

3.1. Theorem. If $G \Rightarrow G^{\prime}$, then $\propto_{G}=\propto_{G^{\prime}}$.

Proof. From $G \Rightarrow G^{\prime}$ it follows that $G^{\prime}=$ $\left\langle N, A, S, T^{\prime}, I\right\rangle$ and there exists an $a \in A$ with $T(a) \in I$ such that equations (1) hold. To prove $\propto_{G}=\propto_{G^{\prime}}$ it is sufficient to prove the following implications:

$p \rightarrow_{G} q \Rightarrow p \propto_{G^{\prime}} q$ for each $p, q \in N$,

$p \rightarrow{ }_{G^{\prime}} q \Rightarrow p \propto_{G} q$ for each $p, q \in N$.

We shall only prove equation (4a), the proof of equation (4b) being similar. Suppose $p \rightarrow_{G} q$ for some $p, q \in N$. Then, $p \in I$ and there exists a $b \in A$ with $S(b)=p$ and $T(b)=q$. If $b \neq a$, then equation (1a) gives $T^{\prime}(b)=q$, so we have $p \rightarrow_{G^{\prime}} q$ and thus $p \alpha_{G^{\prime}} q$. Now consider the case $a=b$. Equation (1b) gives $T^{\prime}(b)=\operatorname{succ}_{G}(q)$, so there exists a $c \in A$ with $S(c)=q$ and $T(c)=T^{\prime}(b)$. If $c=a$, then $c=b$, thus $S(c)=S(b)$ and we obtain $p=q$, which implies $p \propto_{G^{\prime}} q$. Finally, if $c \neq a$, then equation (1a) gives $T^{\prime}(c)=T(c)$, whence $S(c) \rightarrow_{G^{\prime}} T^{\prime}(c)$ becomes $q \rightarrow_{G^{\prime}} T^{\prime}(b)$ and we have $q \propto_{G^{\prime}} T^{\prime}(b)$. Since we also have $p \propto_{G^{\prime}} T^{\prime}(b)$, it follows that $p \propto_{G^{\prime}} q$.

3.2. Theorem. If $G \Rightarrow G^{\prime}$, then $\approx_{G}=\approx_{G^{\prime}}$.

Proof. From $G \Rightarrow G^{\prime}$ it follows that $G^{\prime}=$ $\left\langle N, A, S, T^{\prime}, I\right\rangle$ and there exists an $a \in A$ with $T(a) \in I$ such that equations (1) hold. To prove 
$\approx_{G}=\approx_{G^{\prime}}$ it is sufficient to prove the following equivalence:

$$
\begin{aligned}
& T(b) \propto_{G} T(c) \Leftrightarrow T^{\prime}(b) \propto_{G^{\prime}} T^{\prime}(c) \\
& \forall b, c \in A .
\end{aligned}
$$

We shall only prove the implication from left to right in equation (5), the proof from right to left being similar. First consider the case where $b \neq a$ and $c \neq a$. Then, equation (1a) gives $T(b)=T^{\prime}(b)$ and $T(c)=T^{\prime}(c)$, and thus $T(b) \propto_{G} T(c) \Rightarrow$ $T^{\prime}(b) \propto_{G} T^{\prime}(c)$; Theorem 3.1 now implies $T^{\prime}(b)$ $\propto_{G^{\prime}} T^{\prime}(c)$. The case where $b=a$ and $c=a$ is trivial: $b=c$ implies $T^{\prime}(b) \propto_{G^{\prime}} T^{\prime}(c)$. Finally, consider the case where $c=a$ and $b \neq a$. Equation (1a) gives $T(b)=T^{\prime}(b)$, so $T(b) \propto_{G} T(c)$ implies $T^{\prime}(b) \propto_{G} T(c)$. Equation (1b) gives $T^{\prime}(c)=$ $\operatorname{succ}_{G}(T(c))$, so $T(c) \propto_{G} T^{\prime}(c)$. Together, this gives $T^{\prime}(b) \propto_{G} T^{\prime}(c)$, so from Theorem 3.1 we obtain $T^{\prime}(b) \propto_{G^{\prime}} T^{\prime}(c)$.

From equation (3) it follows that there exists an injective mapping $F$ from equivalence classes of arcs of $G$ to equivalence classes of nodes of $G$ defined by the property that if $x \in A$ and $x$ belongs to the equivalence class $Q$, then $T(x)$ belongs to $F(Q)$.

\subsection{Theorem. The mapping $F$ is invariant under indirection reductions.}

\section{Proof. Suppose}

$G \Rightarrow G^{\prime}=\left\langle N, A, S, T^{\prime}, I\right\rangle$.

$F$ is invariant under indirection reductions if $T(x)$ $\propto_{G} T^{\prime}(x)$ for each $x \in A$ since if $x \in Q$, then $F(Q)$ is the equivalence class containing $T(x)$. There exists an $a \in A$ such that equations (1) hold. From these equations it follows that $T(x) \propto_{G}$ $T^{\prime}(x)$ for each $x \in A$ if $T(a) \propto_{G} \operatorname{succ}_{G}(T(a))$, which is true by definition.

Another useful property is that two equivalent nodes always have a common succ-descendant.

3.4. Theorem. If $m, n \in N$ and $m \propto_{G} n$, then there exists a $p \in N$ such that $m \rightarrow \rightarrow_{G}^{*} p$ and $n \rightarrow{ }_{G}^{*} p$.
Proof. From $m \propto_{G} n$ it follows that there exists a $k \geqslant 1$ and $k$ elements $n_{1}, n_{2}, \ldots, n_{k}$ such that equations (2) hold. The proof is by induction on $k$.

The base step is trivial: $k=1, m=n=p$.

Induction step: suppose $k>1$ and there exists a $p \in N$ such that $m \rightarrow{ }_{G}^{*} p$ and $n_{k-1} \rightarrow_{G}^{*} p$. From equation (2c) we have either $n_{k-1} \rightarrow_{G} n_{k}$ or $n_{k}$ $\rightarrow_{G} n_{k-1}$. If $n_{k} \rightarrow_{G} n_{k-1}$, then $n \rightarrow_{G}^{*} p$, which establishes the desired result. Suppose $n_{k-1} \rightarrow_{G} n_{k}$. If $n_{k-1}=p$, then $m \rightarrow_{G}^{*} n$, which also establishes the desired result. If $n_{k-1} \neq p$, then we have $n_{k-1}$ $\rightarrow_{G} q \rightarrow_{G}^{*} p$ for some $q \in N$ which is only possible if $q=n_{k}$. This proves the theorem.

3.5. Theorem. Each equivalence class of $N$ contains at most one element of $N \backslash I$.

Proof. Suppose $m, n \in N \backslash I$ and $m \propto_{G} n$. From Theorem 3.4 it follows that there exists a $p \in N$ with $m \rightarrow_{G}^{*} p$ and $n \rightarrow_{G}^{*} p$. Thus, $m=p$ and $n=p$, which proves the theorem.

A succ-cycle is a set of elements $c_{1}, c_{2}, \ldots, c_{n}$ of $N$ for some $n \geqslant 1$ with $c_{i} \rightarrow_{G} c_{i+1}$ for all $i=$ $1,2, \ldots, n-1$ and $c_{n} \rightarrow{ }_{G} c_{1}$.

The following lemma is an immediate consequence of our definition of a graph.

Lemma. (a) If $c, c^{\prime} \in N, c$ belongs to some succcycle and $c \rightarrow{ }_{a}^{*} c^{\prime}$, then $c^{\prime}$ also belongs to this succ-cycle.

(b) Two succ-cycles are either equal or disjoint.

(c) All elements of a succ-cycle belong to 1 .

3.6. Theorem. Each equivalence class of $N$ contains at most one succ-cycle.

Proof. Suppose $m, n \in N, m \propto_{G} n$ and both $m$ and $n$ bclong to a succ-cyclc. From Theorem 3.4 it follows that there exists a $p \in N$ with $m \rightarrow{ }_{G}^{*} p$ and $n \rightarrow G_{G}^{*} p$. From part (a) of the Lemma it follows that $p$ belongs to both succ-cycles. From part (b) of the Lemma it then follows that both succ-cycles are equal, which proves the theorem. 
3.7. Theorem. No equivalence class of $N$ contains both an element of $N \backslash I$ and a succ-cycle.

Proof. Suppose $m \in N \backslash I, n \in N, m \propto_{G} n$ and $n$ belongs to a succ-cycle. From Theorem 3.4 it follows that there exists a $p \in N$ with $m \rightarrow_{G}^{*} p$ and $n \rightarrow{ }_{G}^{*} p$. Then $m=p$, since $m \in N \backslash I$, and $p$ belongs to the cycle, due to part (a) of the Lemma. Thus, $m$ belongs to the succ-cycle, which is a contradiction to the fact that $m \in N \backslash I$, due to part (c) of the Lemma. This proves the theorem. $\square$

3.8. Theorem. Each equivalence class of $N$ contains either an element of $N \backslash I$ or a succ-cycle.

Proof. Suppose $m \in N$ and $m$ belongs to an equivalence class $Q$ wich contains no elements of $N \backslash I$. Then we can form the infinite sequence $m_{1}=m$, $m_{2}, \ldots, m_{i}, \ldots$ with $m_{i} \in Q$ and $m_{i} \rightarrow_{G} m_{i+1}$ for all $i \geqslant 1$. Since $Q$ is finite, $Q$ contains a succ-cycle, which proves the theorem.

From Theorems 3.5-3.8 we conclude that the equivalence classes of $N$ can be divided into two types, which we call type $A$ and type $B$ : an equivalence class of type $A$ contains one element of $N \backslash I$ and no succ-cycles; an equivalence class of type B contains one succ-cycle and no elements of $N \backslash I$. We have already seen that equivalence classes are invariant under indirection reductions (see Theorem 3.1). The type of an equivalence class is also invariant under indirection reductions, since the subset $I$ of $N$ is invariant under indirection reductions.

3.9. Theorem. Each equivalence class $Q$ of $N$ contains an element $p \in Q$ such that $n \rightarrow{ }_{G}^{*} p$ for each $n \in Q$. If $Q$ is of type $\mathrm{A}$, then $p \in N \backslash I$; if $Q$ is of type $\mathrm{B}$, then $p$ belongs to the succ-cycle of $Q$.

Pronf. Iet $Q$ he an equivalence class of $N$. For each $n \in Q$, let $Q(n)$ be the subset of $Q$ given by $\left\{n^{\prime} \in Q \mid n^{\prime} \rightarrow_{G}^{*} n\right\}$.

Let $n_{1} \in Q$. If $Q\left(n_{1}\right)=Q$, then $n_{1}$ has the desired properties. Otherwise, let $m$ be an element of
$Q \backslash Q\left(n_{1}\right)$. According to Theorem 3.4 there exists an $n_{2} \in Q$ with $n_{1} \rightarrow_{G}^{*} n_{2}$ and $m \rightarrow_{G}^{*} n_{2}$. For each $n \in Q\left(n_{1}\right)$ we have $n \rightarrow{ }_{G}^{*} n_{1}$ and thus $n \rightarrow_{G}^{*}$ $n_{2}$. Hence, $Q\left(n_{1}\right) \subseteq Q\left(n_{2}\right), m \propto Q\left(n_{1}\right)$, and $m \in$ $Q\left(n_{2}\right)$, so $Q\left(n_{1}\right)$ is a proper subset of $Q\left(n_{2}\right)$. Proceeding in this manner we end up with an $n_{k} \in Q$ with $Q\left(n_{k}\right)=Q$, which proves the existence of a $p \in Q$ with $n \rightarrow \rightarrow_{G}^{*} p$ for each $n \in Q$. If $Q$ is of type $\mathrm{A}$, then there is an $m \in Q$ with $m \in N \backslash I$. From $m \rightarrow{ }_{G}^{*} p$ it then follows that $m=$ $p$. If $Q$ is of type $\mathrm{B}$, then $p \in I, p \rightarrow{ }_{G} \operatorname{succ}_{G}(p)$ and $\operatorname{succ}_{G}(p) \rightarrow \rightarrow_{G}^{*} p$, so $p$ belongs to a succ-cycle. This proves the theorem.

\section{Confluence of indirection reductions}

In this section we shall prove the confluence property for indirection reductions. $G$ will again stand for the graph $\langle N, A, S, T, I\rangle$. The next theorem says that just as a single indirection reduction gives an arc $a$ the new target $\operatorname{succ}_{G}(T(a))$, a series of indirection reductions can give an arc $a$ the new target $n$, where $T(a) \rightarrow_{G}^{*} n$.

4.1. Theorem. Let $a \in A, n \in N$, and $T(a) \rightarrow_{G}^{*} n$. Then there exists a graph $G^{\prime}=\left\langle N, A, S, T^{\prime}, I\right\rangle$ such that $G \Rightarrow^{*} G^{\prime}, T^{\prime}(a)=n$, and $T^{\prime}(x)=T(x)$ for each $x \in A$ with $x \neq a$.

Proof. The proof is done by induction on the lenght of $T(a) \rightarrow_{G}^{*} n$. The base step, where $n=$ $\operatorname{succ}_{G}(T(a))$ is trivial. Suppose $T(a) \rightarrow_{G}^{*} m \rightarrow_{G} n$. By the induction hypothesis there exists a graph $G^{\prime \prime}=\left\langle N, A, S, T^{\prime \prime}, I\right\rangle$ such that $G \Rightarrow^{*} G^{\prime \prime}$, $T^{\prime \prime}(a)=m$, and $T^{\prime \prime}(x)=T(x)$ for each $x \in A$ with $x \neq a$. Suppose $m \rightarrow_{G^{\prime \prime}} n$. Then there exists a graph $G^{\prime}=\left\langle N, A, S, T^{\prime}, I\right\rangle$ with $G^{\prime \prime} \Rightarrow G^{\prime}$, $T^{\prime}(a)=n$, and $T^{\prime}(x)=T^{\prime \prime}(x)$ for each $x \in A$ with $x \neq a$, from which it follows that the claim of the theorem holds. Now consider the case where $m \rightarrow{ }_{G} \prime n$ does not hold. Let $c$ be the unique element of $A$ with $S(c)=m$. Since $T(c)=n$ and $T^{\prime \prime}(c) \neq n$, it follows that $c=a$. Thus, $T(a)=n$, and the claim of the theorem holds with $G^{\prime}=G$. 
$G$ is called a final graph if $a \propto_{G} b \Rightarrow T(a)=$ $T(b)$ for each $a, b \in A$. Each graph can be reduced to a final graph.

4.2. Theorem. There exists a final graph $G^{\prime}$ with $G \Rightarrow^{*} G^{\prime}$.

Proof. Let $Q$ be an equivalence class of $A$. According to Theorem $3.9, F(Q)$ contains a $p$ with $n \rightarrow_{G}^{*} p$ for each $n \in F(Q)$. Let $a \in Q$. According to Theorem 4.1 there exists a graph $G^{\prime}=\left\langle N, A, S, T^{\prime}, I\right\rangle$ such that $G \Rightarrow^{*} G^{\prime}, T^{\prime}(a)$ $=p$, and $T^{\prime}(x)=T(x)$ for each $x \in Q$ and $x \neq a$.

When we show that $n \rightarrow G_{G^{\prime}}^{*} p$ for each $n \in F(Q)$, then by repetition of the above argument for each element of $Q$ there exists a graph $G^{\prime \prime}=$ $\left\langle N, A, S, T^{\prime \prime}, I\right\rangle$ with $G \Rightarrow^{*} G^{\prime \prime}$ and $x \in Q \Rightarrow$ $T^{\prime \prime}(x)=p$; the claim of the theorem then follows by repetition of the above argument for each equivalence class $Q$.

So, let $n$ be an element of $F(Q)$; we shall show that $n \rightarrow G_{G}^{*} p$. Since $n \rightarrow \rightarrow_{G}^{*} p$, there exists a $k \geqslant 1$ and $k$ elements $n_{1}, n_{2}, \ldots, n_{k}$ of $F(Q)$ with $n=n_{1}$, $p=n_{k}$ and $n_{i} \rightarrow_{G} n_{i+1}$ for each $i=1,2, \ldots, k-1$. If $S(a)$ is not in $\left\{n_{i} \mid i=1,2, \ldots, k-1\right\}$, then $n_{i} \rightarrow_{G^{\prime}} n_{i+1}$ for each $i=1,2, \ldots, k-1$ and thus $n \rightarrow G_{G}^{*} p$. If $S(a)=n_{j}$ for some $j$ with $1 \leqslant j \leqslant n-1$ and $S(a)$ is not in $\left\{n_{i} \mid i=1,2, \ldots, j-1\right\}$, then $n_{i} \rightarrow_{G^{\prime}} n_{i+1}$ for each $i=1,2, \ldots, j-1$ and $n_{j} \rightarrow_{G^{\prime}}$ $p$, which implies $n \rightarrow G^{*} p$. This proves the theorem.

We are now able to formulate and prove our main result.

4.3. Theorem. Suppose $G \Rightarrow^{*} G 1$, and $G \Rightarrow^{*} G 2$ for some graphs $G 1$ and $G 2$. Then there exist graphs $G^{\prime}$ and $G^{\prime \prime}$ which are isomorphic such that $G 1 \Rightarrow^{*}$ $G^{\prime}$ and $G 2 \Rightarrow{ }^{*} G^{\prime \prime}$.

Proof. Let $G^{\prime}=\left\langle N, A, S, T^{\prime}, I\right\rangle$ and $G^{\prime \prime}=$ $\left\langle N, A, S, T^{\prime \prime}, I\right\rangle$ be final graphs, $G 1 \Rightarrow^{*} G^{\prime}$ and $G 2 \Rightarrow^{*} G^{\prime \prime}$. Their existence is ensured by Theorem 4.2. From Theorems 3.1 and 3.2 we know that $\propto_{G^{\prime}}=\propto_{G^{\prime \prime}}$ and $\approx_{G^{\prime}}=\approx_{G^{\prime \prime}}$, i.e., $G^{\prime}$ and $G^{\prime \prime}$ have the same equivalence classes of nodes and arcs. From Theorem 3.3 we know that the map- ping $F$ is the same for both graphs. Since both $G^{\prime}$ and $G^{\prime \prime}$ are final graphs, there exists in each equivalence class $Q$ of $N$ elements $p^{\prime}(Q)$ and $p^{\prime \prime}(Q)$ such that $T^{\prime}(a) \in Q$ implies $T^{\prime}(a)=p^{\prime}(Q)$ and $T^{\prime \prime}(a) \in Q$ implies $T^{\prime \prime}(a)=p^{\prime \prime}(Q)$ for each $a \in A$. Note that if $Q$ is of type $\mathrm{A}$, then $p^{\prime}(Q)$ and $p^{\prime \prime}(Q)$ are both equal to the unique element from $N \backslash I$ in $Q$.

In order to prove that $G^{\prime}$ and $G^{\prime \prime}$ are isomorphic we have to show the existence of bijective mappings $f_{N}: N \rightarrow N$ and $f_{A}: A \rightarrow A$ such that

$n \in I \Leftrightarrow f_{N}(n) \in I$,

$f_{N}(S(a))=S\left(f_{A}(a)\right)$ for each $a \in A$,

$f_{N}\left(T^{\prime}(a)\right)=T^{\prime \prime}\left(f_{A}(a)\right)$ for each $a \in A$.

We define $f_{N}$ as follows: on each equivalence class $Q$ of $N, f_{N}$ is a permutation which satisfies $f_{N}\left(p^{\prime}(Q)\right)=p^{\prime \prime}(Q)$. This implies that equation (6) is satisfied.

Now we define $f_{A}$. For each $a \in A$ with $S(a) \neq$ $I, f_{A}(a)$ is equal to $a$, and for each $a \in A$ with $S(a) \in I, f_{A}(a)$ is uniquely determined by $S\left(f_{A}(a)\right)=f_{N}(S(a))$. Obviously, equation (7) is satisfied, and it remains to be shown, that equation (8) is satisfied.

Let $a$ belong to the equivalence class $Q$ of $A$. Then, $T^{\prime}(a)=p^{\prime}(F(Q))$ and thus $f_{N}\left(T^{\prime}(a)\right)=$ $p^{\prime \prime}(F(Q))$. We shall show that $f_{A}(a) \in Q$. Then, $T^{\prime \prime}\left(f_{A}(a)\right)=p^{\prime \prime}(F(Q))$, which proves equation (8). If $S(a) \notin I$, then $f_{A}=a$. If $S(a) \in I$, then $S(a) \propto_{G^{\prime}} T^{\prime}(a)$, and thus $S(a) \in F(Q)$. It follows that $f_{N}(S(a)) \in F(Q)$ and thus $S\left(f_{A}(a)\right) \in F(Q)$. Also, $S\left(f_{A}(a)\right) \in I$, due to equation (6). So, $S\left(f_{A}(a)\right) \propto_{G^{\prime \prime}} T^{\prime \prime}\left(f_{A}(a)\right)$, and thus $T^{\prime \prime}\left(f_{A}(a)\right) \in$ $F(Q)$. It follows that $f_{A}(a) \in Q$, since $F$ is injective. This proves the theorem.

\section{References}

[1] $H$. Ehrig, Introduction to the algebraic theory of graph grammars: A survey, In: V. Claus, H. Ehrig and G. Rozenberg, eds., Graph Grammars and Their Application to Computer Science and Biology, Lecture Notes in Computer Science, Vol. 73 (Springer, Berlin, 1979) 1-69.

[2] H. Ehrig and B.K. Rosen, The mathematics of record handling, SIAM J. Comput. 9 (1980) 411-469. 
[3] S.L. Peyton Jones, The Implementation of Functional Programming Languages (Prentice-Hall, Englewood Cliffs, NJ, 1987).

[4] B.K. Rosen, Tree-manipulating systems and Church-Rosser theorems, J. Assoc. Comput. Mach. 20 (1973) 160-187.

[5] D.A. Turner, A new implementation technique for applica- tive languages, Software - Practice \& Experience 9 (1979) $31-49$.

[6] P.M. Van den Broek and G.F. Van der Hoeven, Indirection Reduction and Garbage Collection in Graph Rewrite Systems, Internal Memorandum, Dept. of Computer Science, Twente Univ., The Netherlands, 1988 , to appear. 